\title{
Immune Responses in Parkinson's Disease: Interplay between Central and Peripheral Immune Systems
}

\author{
Xiaomin Su ${ }^{1}$ and Howard J. Federoff ${ }^{1,2}$ \\ ${ }^{1}$ Department of Neuroscience, Georgetown University Medical Center, Washington, DC 20057, USA \\ ${ }^{2}$ Department of Neurology, Georgetown University Medical Center, Washington, DC 20057, USA
}

Correspondence should be addressed to Howard J. Federoff; hjf8@georgetown.edu

Received 13 February 2014; Accepted 16 March 2014; Published 13 April 2014

Academic Editor: Cheng-Hsien Lu

Copyright (C) 2014 X. Su and H. J. Federoff. This is an open access article distributed under the Creative Commons Attribution License, which permits unrestricted use, distribution, and reproduction in any medium, provided the original work is properly cited.

\begin{abstract}
The etiology of Parkinson's disease (PD) is complex and most likely involves numerous environmental and heritable risk factors. Recent studies establish that central and peripheral inflammation occurs in the prodromal stage of the disease and sustains disease progression. Aging, heritable risk factors, or environmental exposures may contribute to the initiation of central or peripheral inflammation. One emerging hypothesis is that inflammation plays a critical role in PD neuropathology. Increasing evidence suggest that activation of the peripheral immune system exacerbates the discordant central inflammatory response and synergistically drives neurodegeneration. We provide an overview of current knowledge on the temporal profile of central and peripheral immune responses in PD and discuss the potential synergistic effects of the central and peripheral inflammation in disease development. The understanding of the nature of the chronic inflammation in disease progression and the possible risk factors that contribute to altered central and peripheral immune responses will offer mechanistic insights into PD etiology and pathology and benefit the development of effective tailored therapeutics for human PD.
\end{abstract}

\section{Introduction}

Parkinson's disease (PD) is a progressive, age-related, neurodegenerative disorder. Clinically, it is characterized by motor symptoms, such as rigidity, bradykinesia, postural instability, gait disorder, and tremor [1]. However, nonmotor symptoms, such as hyposmia, gastrointestinal abnormalities, and autonomic dysfunction are increasingly accepted as integral parts of PD clinical manifestations and often precede the classical motor symptoms [2]. Pathologically, selective neurodegeneration in the nigrostriatal circuit, presence of dysregulated immune activation, and the occurrence of Lewy bodies (LB) in central and peripheral nervous systems are observed in PD [3]. The cause of nigral neurodegeneration in $\mathrm{PD}$ and its underlying mechanisms remain elusive; however, involvement of inflammatory events has been postulated to contribute to neuronal loss. Indeed, inflammation has been linked to many other age-related chronic neurodegenerative diseases, including Alzheimer's disease [4], amyotrophic lateral sclerosis $[5,6]$ and Huntington's disease $[7,8]$. Triggering factors of inflammation may be dysregulation of inflammatory pathways (e.g., immune alteration associated with aging or genetic vulnerability), pathogens (e.g., bacterial or viral infection), environmental toxins (e.g., pesticides), and protein aggregates (e.g., $\alpha$-synuclein $(\alpha$-Syn)).

There has been considerable debate in the field as to whether inflammation is a driving force in neurodegeneration or simply represents a response to neuronal death. Here, we describe the temporal profile of altered immune responses, including central and peripheral inflammation in the disease progression, present evidence indicating the interaction between the central, and peripheral inflammation in both sporadic and familial PD, and discuss recent data supporting the key role of inflammatory responses in the initiation and progression of disease pathology.

\section{Central Inflammation in PD}

Microglia-associated central inflammation is a pathological hallmark of PD. Initial evidence of the involvement of 
inflammation in the progression of PD stems from a postmortem study over twenty years ago, which demonstrated the presence of activated microglia in the substantia nigra pars compacta $(\mathrm{SNpc})$ of a $\mathrm{PD}$ patient [9]. Since then, an abundance of clinical and animal studies supports the role of activated microglia and increased levels of inflammatory mediators such as cytokines, chemokines, and ROS in the pathology of PD [10-14].

The central inflammatory process (activation/proliferation of microglia/ astrocyte and secretion of proinflammatory cytokines and free radicals) could be an event secondary to the neurodegenerative process and, in turn, exacerbates the progression of cell death. However, under certain circumstances, inflammation could be a primary event that leads to the neurodegenerative process. A clinical study that uses positron emission tomography to study activated microglia in the brain of idiopathic PD patients shows the absence of significant longitudinal changes in microglia activation over time suggesting that brain inflammation occurs early in PD process [15]. We observe early inflammation in transgenic mice with overexpression or mutation (A53T and A30P) of $\alpha$-Syn, characterized by microglial activation and increased expression in proinflammatory cytokines, prior to dopaminergic neuronal cell death and motor disorder [16, 17]. Another study using intranigral injection of lipopolysaccharide (LPS) on the dopaminergic system of the rat shows that LPS initially induces microglial activation in a short time (within 2 days) and subsequently causes a progressive degeneration of the dopaminergic system in the long term (up to one year after LPS injection) [18], suggesting that microglia-mediated inflammation underlies the neuronal cell death in the SN.

Activated microglia exert their neurotoxic effects by releasing proinflammatory cytokines such as TNF $\alpha$, IL- $1 \beta$, IL-6, and IFN $\gamma$ and free radicals including ROS and NO, as well as inflammatory mediators such as prostaglandins E2 (PGE2), leading to nigral cell damage and death. Enhanced expression of TNF $\alpha$, IL- $1 \beta$, IL- 6 , and INF $\gamma$ has been shown in basal ganglia as well as cerebrospinal fluid of $\mathrm{PD}$ patients [19-21]. TNF $\alpha$ and IL- $1 \beta$ are robust activators of NF- $\kappa$ B and contribute to neuronal cell death by triggering apoptotic transduction pathway [22-24]. Additionally, TNF $\alpha$, IL-1 $\beta$, and INF $\gamma$ induce potent activation of iNOS [25], presumably mediated by a low-affinity IgE receptor CD23, which is expressed exclusively on glial cells in the SN of PD patients [26]. iNOS is responsible for NO production, contributing to neuronal toxicity [27]. Collectively, cytokine/CD23dependent activation of iNOS in microglia may be involved in the cascade of events leading to dopaminergic neuronal degeneration [28]. Moreover, TNF $\alpha$ and IL-1 $\beta$ can upregulate COX2, resulting in the production of PGE2 and induction of an intraneuronal toxic effect directly on dopaminergic neurons [29-31]. Given that dopaminergic neurons in the SN are relatively sensitive to "stress" [32] and that there is a large population of microglia in the $\mathrm{SN}$ in comparison to other CNS regions [33], inflammation is a crucial step in the pathogenic cascade leading to neurodegeneration.

Central inflammation pertains to all forms of PD, both genetic and idiopathic. Aging is a major factor for both genetic and idiopathic PD. Recent evidence suggests that aged brain resides in a chronic state of neuroinflammation, characterized by increased reactivity upon immune stimulation and low-grade production and central cytokines [18, 34-36]. One working hypothesis suggests that this hyperreactivity is due to priming of brain microglial cells. Microglia in aged brains become "primed," exhibiting increased expression of MHC class II, scavenger receptor CD68 [37, 38], CD11b, and CD11c integrins [34, 39], Toll-like receptor TLR4 [40], and costimulatory molecule CD86 [41] and being capable of adopting a potent neurotoxic and proinflammatory phenotype [42]. Subsequent to peripheral innate immune stimulation, microglia in aged brains respond with an exaggerated inflammatory response compared to younger cohort [37, 43]. The precise priming stimulus of aging has yet to be identified. Some studies have suggested that IFN- $\gamma$ concentrations are increased in the aged brain [44], which may implicate IFN- $\gamma$ as a candidate molecule for microglial priming. To this end, the treatment of human microglial cell cultures with IFN$\gamma$ results in microglial activation, as evidenced by increased production of reactive oxygen species [45]. This scheme resembles the activation process of peripheral macrophages, wherein the classical macrophage activating factor, produced by stimulated Th1 lymphocytes and NK cells, is IFN- $\gamma$ [46]. Other studies indicate that aging is associated with a reduction in anti-inflammatory cytokines including IL-10 and IL-4 $[47,48]$.

Astrocytes play equally important immunomodulatory role in maintaining CNS homeostasis. Altered astrocytic function is now recognized as a primary contributing factor to an increasing number of neurological diseases. With age, astrocytes have a more inflammatory profile. For instance, there is increased expression of astrocytic glial fibrillary acidic protein (GFAP) in the brain of aged rodents and humans $[49,50]$. In addition, vimentin, an intermediate filament protein, also increases with aging in humans [51]. The age-related increases in GFAP and vimentin are similar to the activated astrocytic profile associated with inflammation and traumatic CNS injury [52]. There are many potential consequences of a more inflammatory astrocyte in the aged brain. First, astrocytes communicate directly with microglia, so an inflammatory astrocyte phenotype may elevate the amplitude and duration of microglia-mediated neuroinflammation [53]. Second, astrocytes play the most substantial role in maintaining an intact blood-brain barrier (BBB) [54]. Age-related changes in astrocytes can affect BBB permeability, especially under inflammatory conditions and neurodegenerative diseases $[5,55]$. Third, astrocytes secrete chemokine monocyte chemoattractant protein-1 (MCP-1) which is a key chemokine involved in the recruitment of peripheral monocytes [56]. In summary, recent studies have shed some light on astrocyte-mediated neuroinflammation in neurodegeneration, and future research on astrocyte pathophysiology is expected to provide new perspectives on neurodegeneration and potential therapeutic strategies.

Several PD-linked genetic mutations are associated with increased glial activation in mediating chronic PD progression. A genetic dysfunction of $\alpha$-Syn coupled with increased neuroinflammation can potentiate each other, driving chronic progression of neurodegeneration. Increasing 
experimental findings point to clear roles for $\alpha$-Syn in the central inflammation in PD: (I) overexpression or mutation of $\alpha$-Syn in the dopaminergic neurons leads to neuroinflammatory responses in $\alpha$-Syn transgenic animals [16, 17]; (II) direct intranigral injection of $\alpha$-Syn results in the production of proinflammatory cytokines and microglial activation in mouse brain [57]; (III) in the MPTP mouse model, nitrated form of $\alpha$-Syn (N- $\alpha$-Syn) is shown to drain into cervical lymph nodes and to elicit an antigen-specific $\mathrm{T}$ cell response. $\mathrm{N}-\alpha$-Syn specific effector T cells exacerbate microglial activation and DA neurodegeneration [58, 59]. Collectively $\alpha$-Syn in SN DA neurons may elicit a selfpropelling cycle of microglial activation and overproduction of inflammatory mediators in $\mathrm{SN}$, leading to $\mathrm{PD}$-associated dysfunction and spreading to neighboring neurons [60]. The mechanisms by which $\alpha$-Syn initiates central inflammation remain to be determined. Recent data support the hypothesis that $\alpha$-Syn is released from neurons into the interstitial space where the protein would be available to directly stimulate microglial activation via the scavenger receptor CD36 and the prostaglandin E2 receptor subtype 2 (EP2) $[16,17,61,62]$.

Mutations in Leucine-rich repeat kinase 2 (LRRK2) contribute to both idiopathic and familial forms of PD. LRRK2 expression is readily detected in multiple immune cells including B-lymphocytes, monocytes, dendritic cells, and microglial $[63,64]$, which suggests a potential role for LRRK2 in the immune system. PD-linked LRRK2 mutation (R1441G) increases proinflammatory cytokine release from activated primary microglial cells [65]. Furthermore, LRRK2 R1441G stabilizes cyclooxygenase 2 RNA and increases inflammatory response in idiopathic and genetic PD fibroblast [66]. In addition, LRRK2 in microglia plays a key role in the phagocytosis of neuronal elements [67]. In contrast, LRRK2 deficiency attenuates LPS-induced mRNA and/or protein expression of inducible nitric oxide synthase, TNF- $\alpha$, IL-1 $\beta$, and IL-6 [68]. Taken together, these results support LRRK2 as a positive regulator of inflammation in microglia, and disease-related LRRK2 mutations may shift the microenvironment of the brain to favor neuroinflammation.

Loss-of-function mutations in the E3 ligase Parkin give rise to a rare form or autosomal recessive parkinsonism [69]. Although mice deficient in Parkin do not display nigral degeneration, chronic administration of low-dose LPS trigger very similar neuroinflammatory and oxidative stress responses in the SNpc of both WT and Parkindeficient mice; only the latter develops delayed and selective degeneration of DA neurons in SNpc but not in VTA [70]. These findings suggest that Parkin loss changes sensitivity to specific inflammatory mediators and increases vulnerability to inflammation-induced degeneration. Additional studies will need to establish whether and how Parkin-deficient glia is "primed" and respond aberrantly to exacerbate neurodegeneration.

The altered level or activity of certain gene products in CNS cells may contribute to central inflammatory response as well, although the mutation of these genes has not been identified in familial PD. For instance, the GPEX consortium reports a PD meta-analysis of gene expression data indicating that the mitochondrial master regulator, peroxisome proliferator-activated receptor gamma coactivator-1 alpha, PGC- $1 \alpha$, and related bioenergetic genes, including those encoding NADH ubiquinone oxidase (Complex I), succinate dehydrogenase (Complex II), cytochrome C oxidase (Complex IV), and ATP synthase (Complex V), are down-regulated in affected brain tissue from patients with both symptomatic and subclinical PD [71]. This observation suggests that PGC- $1 \alpha$ is emerging as a molecular link between mitochondrial dysfunction and transcriptional dysregulation in PD. In vivo studies have shown that PGC- $1 \alpha$ knockouts are much more sensitive to the neurodegenerative effects of MPTP, and increased PGC- $1 \alpha$ levels protect neurons from oxidative stress in vitro, $\alpha$-Syn-mediated cell death in vitro, and MPTP-mediated neuronal degeneration in vivo $[72,73]$. Interestingly, a long-term study using muscle-specific PGC$1 \alpha$ knockout mice demonstrates that loss of muscle PGC$1 \alpha$ causes age-dependent low-grade, chronic inflammation in white adipose and liver tissue [74]. Whether PGC- $1 \alpha$ deficiency in CNS cells will equally cause age-dependent low-grade inflammation in the brain remains unknown but warrant further investigation. Understanding the role of PGC- $1 \alpha$ in the central as well as peripheral immune responses will provide new perspective for PD treatment.

\section{Peripheral Inflammation in PD}

The link between peripheral inflammation and neurodegeneration in PD patients has been revealed in several clinical reports. The clinical evidence for systemic inflammation in PD includes the presence of elevated serum levels of TNF $\alpha$ and $\mathrm{TNF} \alpha$ receptor 1 in $\mathrm{PD}$ patients compared to control subjects $[14,75,76]$. Also, elevated plasma concentrations of IL- 6 correlate with the increased risk of PD [77]. In addition, gut inflammation occurs in PD patients [78-80]. PD patients often suffer infectious disease, and the main causes of death are pneumonia and respiratory infections [81-84]. Furthermore, cytotoxic T lymphocyte (CD4+ and CD8+) has been described to infiltrate the SN of PD patients $[85,86]$. The influx of these peripheral cells into the brain parenchyma could indicate a BBB dysfunction in PD patients $[87,88]$.

The association of peripheral inflammation and $\mathrm{PD}$ pathogenesis is further demonstrated in PD animal models. Pregnant rats exposed to intraperitoneal (i.p.) injection of LPS resulted in a decreased number of dopaminergic neurons in the pups when compared to nonexposed controls [89]. Similarly, rat fetuses exposed to LPS are more susceptible to 6 -OHDA in adulthood $[90,91]$. In adult animals, there is also data that strongly suggests the role of peripheral inflammation in the ongoing PD model. Animals with an increased peripheral inflammatory response after bacterial LPS injection are associated with central dopaminergic hypoactivity [92]. Peripheral inflammation induced by ulcerative colitis worsens the effects induced by intranigral LPS, including dopaminergic neuronal cell loss, microglial inflammation, and alteration in $\mathrm{BBB}$ permeability [93]. All previous data indicate a close relationship between the peripheral immune system and the central dopaminergic system. 


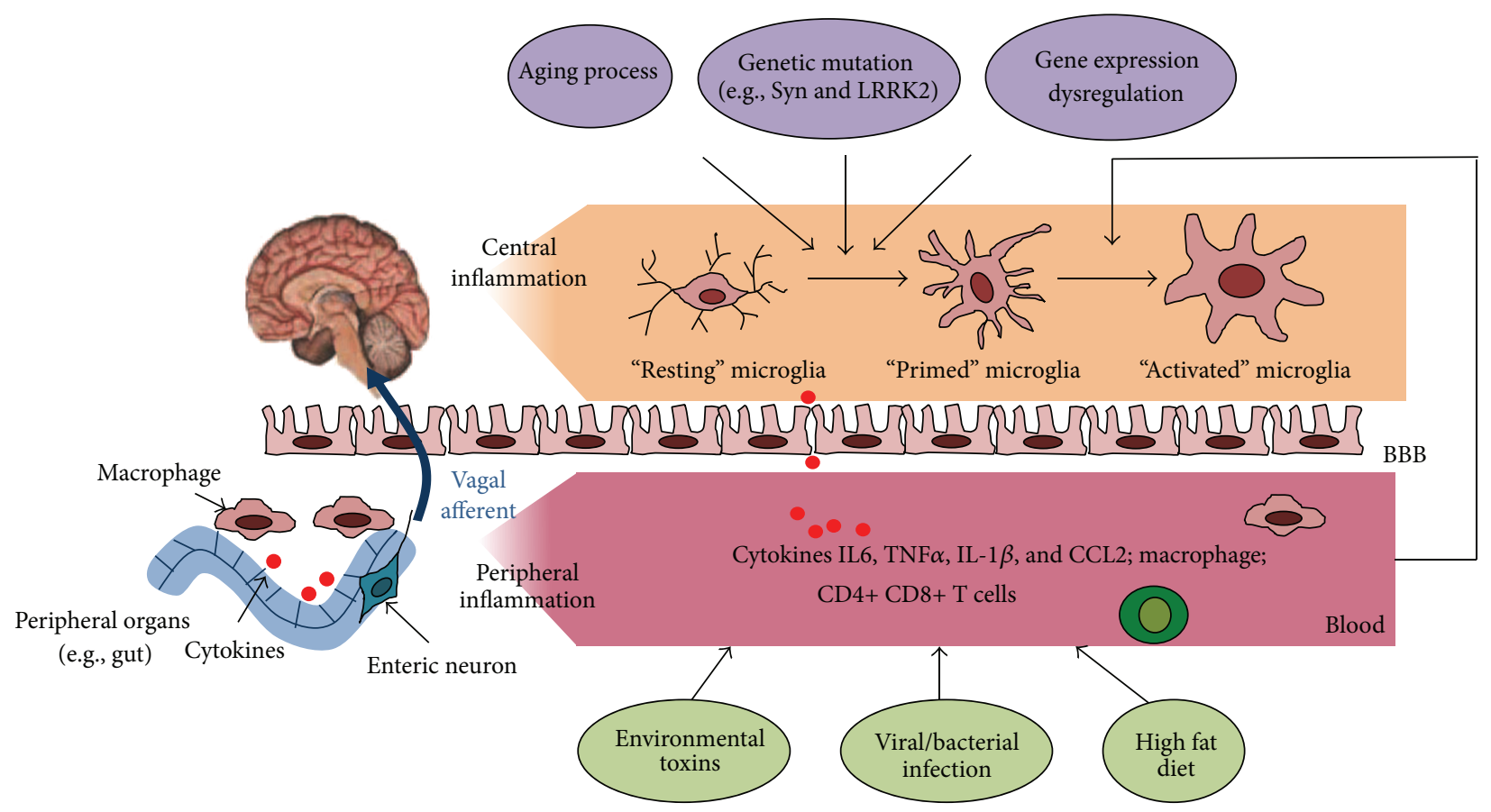

FIGURE 1: Interplay between central and peripheral immune systems in Parkinson's disease. The aging process, genetic mutation, and/or dysregulation of certain gene expressions serve as a "priming" stimulus for microglia. Upon secondary stimulation (e.g., environmental toxin, viral infection, high fat diet), peripheral inflammation is induced and communicates with brain through neural (vagal afferent) or humoral routs (e.g., cytokines circulation). The primed microglia are further activated and release excessive quantities of proinflammatory cytokines driving neurodegeneration.

It becomes more clear that peripheral inflammation plays a role in early stages of disease initiation and progression, including the development of preclinical nonmotor symptoms (hyposmia, constipation, bladder disorder, sleep disorder, obesity, and depression; Braak stages I and II [94]). Peripheral inflammation appears to accompany the nonmotor symptom of PD. One clinical study observes a significant correlation between serum $\mathrm{TNF} \alpha$ levels and the severity of nonmotor symptoms including depression, sleep disturbances, and cognitive dysfunction in PD patients [95]. Gut infection and inflammation, mediated by a gramnegative bacterium, Helicobacter pylori (HP), are associated with PD [96, 97]. Moreover, successful treatment of HP has been shown to increase stride length in PD patients, whereas failure to eradicate HP results in significantly worse symptoms [98]. Chronic constipation, which occurs as early as 20 or more years before the onset of motor symptoms of $\mathrm{PD}$, is casually linked to peripheral inflammation. Stomach infections may have early consequences on the enteric nervous system that manifest as gastrointestinal (GI) dysfunction including constipation [99]. Obesity, which is associated with increased risk of developing PD [100], displays increased levels of proinflammatory cytokines including TNF $\alpha$, IL-1 $\beta$, IL6 , and CCL2, in adipose tissue [101-103], liver [104], pancreas [105], brain [106], and possibly muscle [107]. Other studies have demonstrated that anti-inflammatory therapy may ameliorate MNS in non-PD related condition [108]. Collectively, strong clinical data support that peripheral inflammation appears to be an early event in the development of PD.

\section{Interplay of Central and Peripheral Systems Drives Neurodgeneration}

The CNS has been considered as immunologically privileged and protected by the BBB which prevents entry of pathogens and immune cells into the parenchyma. However, recent evidence suggests that the communication between central CNS and periphery is very fluid. BBB breakdown and systemic inflammation appear to play an important role in the pathology of numerous neurodegenerative diseases compromising the vascular unit and inducing leukocyte migration within the brain parenchyma [109]. A systemic infection or injury gives rise to an inflammatory response that communicates with brain. Both neural and humoral routes mediate communication from the site of peripheral inflammation to the brain. The neural route is through the dorsal motor nucleus of the vagus nerve [110]. The humoral route involves circulating cytokines that communicate with the brain via several routes: (i) by saturable transport across the BBB [111]; (ii) by activating endothelial cells and perivascular macrophages [112]; and (iii) through circumventricular organs which lack a functional BBB [113].

Recent studies suggest that activation of the peripheral immune systems exacerbates the discordant central inflammatory response in aged or genetic predisposed brains. For instance, LPS challenge promotes microglial hyperactivity in aged mice, that is, associated with exaggerated induction of both proinflammatory ILlbeta and anti-inflammatory IL10 cytokines [114]. In addition, Gao et al. have established 
a two-hit animal model involving $\alpha$-Syn mutation (A53T) and an environmental trigger (LPS) [115], which reproduces key features of PD and demonstrates synergistic effects of genetic predisposition and environmental exposures in the development of PD. Collectively, the aging process, genetic mutation, and/or dysregulation of certain gene expression serve as a "priming" stimulus for microglia, and upon secondary stimulation (e.g., environmental toxin or viral infection), the primed microglia release excessive quantities of proinflammatory cytokines driving neurodegeneration (Figure 1).

\section{Therapeutic Implication}

We are at the beginning of understanding the impact of chronic inflammation inside and outside of the brain towards neurodegenerative disorders. The more we are certain about these interactions, the better we would be able to diagnose, manage, and treat Parkinson's disease in a systemic but targeted manner.

Chronic inflammation in both sporadic and familial PD may represent therapeutic opportunities for immunomodulatory interventions in combination with other neuroprotective agents. However, the negative results of nonsteroidal anti-inflammatory drugs in late PD [116] strongly suggest that early immunomodulation is the key in preventing PD onset and progression. Minocycline, a broad-spectrum tetracycline antibiotic, has been tested in experimental models and PD patients. Minocycline effectively crosses the BBB and shows potent anti-inflammatory and neuroprotective effects in neurotoxin models of PD (e.g., MPTP and rotenone) [117]. A randomized, double-blind, Phase II futility clinical trial shows that minocycline offers clinical benefit to early PD patients, which warrants further consideration of minocycline for Phase III clinical trials [118]. Pioglitazone, a synthetic peroxisome proliferator-activated receptor gamma (PPAR- $\gamma$ ) agonist, is currently under investigation in a Phase II placebocontrolled clinical trial for the treatment of early PD.

LRRK2 is highly expressed in peripheral macrophage and monocytic cells as well as in central microglia, suggesting a functional role for LRRK2 in the innate immune system [119, 120]. Recent studies show that LRRK2 kinase inhibitors attenuate inflammatory signaling in HIV or LPS-treated microglia $[67,120]$. Furthermore, small hairpin RNA targeting LRRK2 can equally inhibit LPS-induced microglial activation [120]. These data strongly suggest that inhibition or attenuation of LRRK2 is a promising therapeutic strategy for antiinflammatory treatment for PD. However, it should be noted that LRRK2 knockout mice display alterations in exploratory and motor coordination behaviors and cause degeneration in the kidney [121], suggesting the wild-type LRRK2 may be involved in certain important normal physiological functions. Thus, a safe and effective LRRK2 therapeutic strategy using small molecule inhibitors or RNA interference should be specific and target disease-linked mutations.

PGC- $1 \alpha$ is a potential new target for anti-inflammatory therapy for PD. Several pharmacological activators have been reported to enhance PGC-1a activity and stimulate mitochondrial biogenesis. PGC-la activity is mainly controlled by the PPARs, AMPK, and Sirtl [122]. Hence, pharmacological activators for these proteins have the potential to exert anti-inflammation as well as induce mitochondrial biogenesis through PGC- $1 \alpha$ activation. Such activators include fibrates and rosiglitazone (PPAR) [123, 124], metformin [125], pyrroloquinoline quinone (PQQ) [126], and AICAR [127] (AMPK) as well as resveratrol (Sirtl) [128]. As PPAR agonist (fibrates, rosiglitazone) and AMPK activator (metformin, AICAR) are already routinely used in clinical practice for treatment of metabolic syndrome and Type 2 Diabetes, these drugs could be readily translated from animal models to PD patients. Preclinical CNS distribution and efficacy studies using inflammatory animal models of PD (i.e., the two-hit model) will be sufficient to warrant clinical trials on these drugs.

\section{Conclusions}

Although PD is complex multifactorial disorder with unknown etiology, increasing evidence supports an important role of central and peripheral inflammation in driving $\mathrm{PD}$ initiation and progression. Therefore, the current critical need is to identify promising targets for anti-inflammatory therapies, as well as fully understand the potential effects, both positive and negative, of blocking the inflammatory state in the early stage of the disease. Understanding the link between PD genetic variants or altered transcription and specific immune responses is crucial to identify novel therapeutic targets and to devise tailored neuroprotective interventions. As the research on preclinical/subclinical biomarkers for PD advances, antiinflammation therapies clinical trials will become feasible for those at highest risk for PD.

\section{Conflict of Interests}

The authors declare that there is no conflict of interests regarding the publication of this paper.

\section{References}

[1] I. Litvan, K. P. Bhatia, D. J. Burn et al., "SIC task force appraisal of clinical diagnostic criteria for parkinsonian disorders," Movement Disorders, vol. 18, no. 5, pp. 467-486, 2003.

[2] D. W. Dickson, H. Fujishiro, C. Orr et al., "Neuropathology of non-motor features of Parkinson disease," Parkinsonism and Related Disorders, vol. 15, 3, pp. S1-S5, 2009.

[3] W. Dauer and S. Przedborski, "Parkinson's disease: mechanisms and models," Neuron, vol. 39, no. 6, pp. 889-909, 2003.

[4] A. R. Kamer, R. G. Craig, A. P. Dasanayake, M. Brys, L. GlodzikSobanska, and M. J. de Leon, "Inflammation and Alzheimer's disease: possible role of periodontal diseases," Alzheimer's and Dementia, vol. 4, no. 4, pp. 242-250, 2008.

[5] B. O. Popescu, E. C. Toescu, L. M. Popescu et al., "Bloodbrain barrier alterations in ageing and dementia," Journal of the Neurological Sciences, vol. 283, no. 1-2, pp. 99-106, 2009. 
[6] M. C. Evans, Y. Couch, N. Sibson, and M. R. Turner, "Inflammation and neurovascular changes in amyotrophic lateral sclerosis," Molecular and Cellular Neurosciences, vol. 53, pp. 3441, 2013.

[7] F. Sanchez-Lopez, I. Tasset, E. Aguera et al., "Oxidative stress and inflammation biomarkers in the blood of patients with Huntington's disease," Neurological Research, vol. 34, no. 7, pp. 721-724, 2012.

[8] H. Y. Hsiao, Y. C. Chen, H. M. Chen, P. H. Tu, and Y. Chern, "A critical role of astrocyte-mediated nuclear factor-kappaBdependent inflammation in Huntington's disease," Human Molecular Genetics, vol. 22, no. 9, pp. 1826-1842, 2013.

[9] P. L. McGeer, S. Itagaki, B. E. Boyes, and E. G. McGeer, "Reactive microglia are positive for HLA-DR in the substantia nigra of Parkinson's and Alzheimer's disease brains," Neurology, vol. 38, no. 8, pp. 1285-1291, 1988.

[10] D. Blum-Degena, T. Müller, W. Kuhn, M. Gerlach, H. Przuntek, and P. Riederer, "Interleukin- $1 \beta$ and interleukin- 6 are elevated in the cerebrospinal fluid of Alzheimer's and de novo Parkinson's disease patients," Neuroscience Letters, vol. 202, no. 1-2, pp. 17-20, 1995.

[11] M. Mogi, M. Harada, T. Kondob et al., "Interleukin-1 $\beta$, interleukin-6, epidermal growth factor and transforming growth factor- $\alpha$ are elevated in the brain from parkinsonian patients," Neuroscience Letters, vol. 180, no. 2, pp. 147-150, 1994.

[12] M. Mogi, M. Harada, P. Riederer, H. Narabayashi, K. Fujita, and T. Nagatsu, "Tumor necrosis factor- $\alpha$ (TNF- $\alpha$ ) increases both in the brain and in the cerebrospinal fluid from parkinsonian patients," Neuroscience Letters, vol. 165, no. 1-2, pp. 208-210, 1994.

[13] M. Mogi, M. Harada, H. Narabayashi, H. Inagaki, M. Minami, and T. Nagatsu, "Interleukin (IL)-1 $\beta$, IL-2, IL-4, IL-6 and transforming growth factor- $\alpha$ levels are elevated in ventricular cerebrospinal fluid in juvenile parkinsonism and Parkinson's disease," Neuroscience Letters, vol. 211, no. 1, pp. 13-16, 1996.

[14] M. Reale, C. Iarlori, A. Thomas et al., "Peripheral cytokines profile in Parkinson's disease," Brain, Behavior, and Immunity, vol. 23, no. 1, pp. 55-63, 2009.

[15] A. Gerhard, N. Pavese, G. Hotton et al., "In vivo imaging of microglial activation with [11C](R)-PK11195 PET in idiopathic Parkinson's disease," Neurobiology of Disease, vol. 21, no. 2, pp. 404-412, 2006.

[16] X. Su, K. A. Maguire-Zeiss, R. Giuliano, L. Prifti, K. Venkatesh, and H. J. Federoff, "Synuclein activates microglia in a model of Parkinson's disease," Neurobiology of Aging, vol. 29, no. 11, pp. 1690-1701, 2008.

[17] X. Su, H. J. Federoff, and K. A. Maguire-Zeiss, "Mutant $\alpha$-synuclein overexpression mediates early proinflammatory activity," Neurotoxicity Research, vol. 16, no. 3, pp. 238-254, 2009.

[18] A. Castaño, A. J. Herrera, J. Cano, and A. Machado, "Lipopolysaccharide intranigral injection induces inflammatory reaction and damage in nigrostriatal dopaminergic system," Journal of Neurochemistry, vol. 70, no. 4, pp. 1584-1592, 1998.

[19] S. Hunot, F. Boissière, B. Faucheux et al., "Nitric oxide synthase and neuronal vulnerability in Parkinson's disease," Neuroscience, vol. 72, no. 2, pp. 355-363, 1996.

[20] E. C. Hirsch, S. Hunot, P. Damier, and B. Faucheux, "Glial cells and inflammation in Parkinson's disease: a role in neurodegeneration?” Annals of Neurology, vol. 44, no. 3, pp. S115-S120, 1998.
[21] T. Nagatsu, M. Mogi, H. Ichinose, and A. Togari, "Cytokines in Parkinson's disease," Journal of Neural Transmission, no. 58, pp. 143-151, 2000.

[22] M. Mogi, A. Togari, T. Kondo et al., "Caspase activities and tumor necrosis factor receptor R1 (p55) level are elevated in the substantia nigra from Parkinsonian brain," Journal of Neural Transmission, vol. 107, no. 3, pp. 335-341, 2000.

[23] N. P. Hailer, C. Vogt, H.-W. Korf, and F. Dehghani, "Interleukin$1 \beta$ exacerbates and interleukin-1 receptor antagonist attenuates neuronal injury and microglial activation after excitotoxic damage in organotypic hippocampal slice cultures," European Journal of Neuroscience, vol. 21, no. 9, pp. 2347-2360, 2005.

[24] C. Li, J. Zienkiewicz, and J. Hawiger, "Interactive sites in the MyD88 Toll/interleukin (IL) 1 receptor domain responsible for coupling to the IL1 $\beta$ signaling pathway," Journal of Biological Chemistry, vol. 280, no. 28, pp. 26152-26159, 2005.

[25] A. Hartmann, S. Hunot, and E. C. Hirsch, "Inflammation and dopaminergic neuronal loss in Parkinson's disease: a complex matter," Experimental Neurology, vol. 184, no. 2, pp. 561-564, 2003.

[26] S. Hunot, N. Dugas, B. Faucheux et al., "FceRII/CD23 is expressed in Parkinson's disease and induces, in vitro, production of nitric oxide and tumor necrosis factor- $\alpha$ in glial cells," Journal of Neuroscience, vol. 19, no. 9, pp. 3440-3447, 1999.

[27] V. L. Dawson, T. M. Dawson, D. A. Bartley, G. R. Uhl, and S. H. Snyder, "Mechanisms of nitric oxide-mediated neurotoxicity in primary brain cultures," Journal of Neuroscience, vol. 13, no. 6, pp. 2651-2661, 1993.

[28] S. Hunot and R. A. Flavell, "Death of a monopoly?" Science, vol. 292, no. 5518, pp. 865-866, 2001.

[29] H.-M. Gao, B. Liu, W. Zhang, and J.-S. Hong, "Novel antiinflammatory therapy for Parkinson's disease," Trends in Pharmacological Sciences, vol. 24, no. 8, pp. 395-401, 2003.

[30] Z. Li, M. Jansen, K. Ogburn et al., "Neurotoxic prostaglandin $\mathrm{J} 2$ enhances cyclooxygenase- 2 expression in neuronal cells through the p38MAPK pathway: a death wish?" Journal of Neuroscience Research, vol. 78, no. 6, pp. 824-836, 2004.

[31] S. G. Harris, J. Padilla, L. Koumas, D. Ray, and R. P. Phipps, "Prostaglandins as modulators of immunity," Trends in Immunology, vol. 23, no. 3, pp. 144-150, 2002.

[32] V. Jackson-Lewis and R. J. Smeyne, "MPTP and SNpc DA neuronal vulnerability: role of dopamine, superoxide and nitric oxide in neurotoxicity. Minireview," Neurotoxicity Research, vol. 7, no. 3, pp. 193-201, 2005.

[33] M. L. Block, L. Zecca, and J.-S. Hong, "Microglia-mediated neurotoxicity: uncovering the molecular mechanisms," Nature Reviews Neuroscience, vol. 8, no. 1, pp. 57-69, 2007.

[34] V. H. Perry, M. K. Matyszak, and S. Fearn, "Altered antigen expression of microglia in the aged rodent CNS," Glia, vol. 7, no. 1, pp. 60-67, 1993.

[35] L. G. Sheffield and N. E. J. Berman, "Microglial expression of MHC class II increases in normal aging of nonhuman primates," Neurobiology of Aging, vol. 19, no. 1, pp. 47-55, 1998.

[36] M. G. Frank, R. M. Barrientos, J. C. Biedenkapp, J. W. Rudy, L. R. Watkins, and S. F. Maier, "mRNA up-regulation of MHC II and pivotal pro-inflammatory genes in normal brain aging," Neurobiology of Aging, vol. 27, no. 5, pp. 717-722, 2006.

[37] J. P. Godbout, J. Chen, J. Abraham et al., "Exaggerated neuroinflammation and sickness behavior in aged mice following activation of the peripheral innate immune system," The FASEB Journal, vol. 19, no. 10, pp. 1329-1331, 2005. 
[38] A. M. Wong, N. V. Patel, N. K. Patel et al., "Macrosialin increases during normal brain aging are attenuated by caloric restriction," Neuroscience Letters, vol. 390, no. 2, pp. 76-80, 2005.

[39] C. C. Stichel and H. Luebbert, "Inflammatory processes in the aging mouse brain: participation of dendritic cells and T-cells," Neurobiology of Aging, vol. 28, no. 10, pp. 1507-1521, 2007.

[40] M. Letiembre, W. Hao, Y. Liu et al., "Innate immune receptor expression in normal brain aging," Neuroscience, vol. 146, no. 1, pp. 248-254, 2007.

[41] R. Griffin, R. Nally, Y. Nolan, Y. McCartney, J. Linden, and M. A. Lynch, "The age-related attenuation in long-term potentiation is associated with microglial activation," Journal of Neurochemistry, vol. 99, no. 4, pp. 1263-1272, 2006.

[42] E. G. Njie, E. Boelen, F. R. Stassen, H. W. M. Steinbusch, D. R. Borchelt, and W. J. Streit, "Ex vivo cultures of microglia from young and aged rodent brain reveal age-related changes in microglial function," Neurobiology of Aging, vol. 33, no. 1, pp. 195.e1-195.e12, 2012.

[43] J. Chen, J. B. Buchanan, N. L. Sparkman, J. P. Godbout, G. G. Freund, and R. W. Johnson, "Neuroinflammation and disruption in working memory in aged mice after acute stimulation of the peripheral innate immune system," Brain, Behavior, and Immunity, vol. 22, no. 3, pp. 301-311, 2008.

[44] F. O. Maher, R. M. Clarke, A. Kelly, R. E. Nally, and M. A. Lynch, "Interaction between interferon $\gamma$ and insulin-like growth factor-1 in hippocampus impacts on the ability of rats to sustain long-term potentiation," Journal of Neurochemistry, vol. 96, no. 6, pp. 1560-1571, 2006.

[45] C. C. Chao, S. Hu, and P. K. Peterson, "Modulation of human microglial cell superoxide production by cytokines," Journal of Leukocyte Biology, vol. 58, no. 1, pp. 65-70, 1995.

[46] D. R. Davila, C. K. Edwards III, S. Arkins, J. Simon, and K. W. Kelley, "Interferon- $\boldsymbol{\gamma}$-induced priming for secretion of superoxide anion and tumor necrosis factor- $\alpha$ declines in macrophages from aged rats," The FASEB Journal, vol. 4, no. 11, pp. 2906-2911, 1990.

[47] F. O. Maher, Y. Nolan, and M. A. Lynch, "Downregulation of IL4 -induced signalling in hippocampus contributes to deficits in LTP in the aged rat," Neurobiology of Aging, vol. 26, no. 5, pp. 717-728, 2005.

[48] S.-M. Ye and R. W. Johnson, "An age-related decline in interleukin-10 may contribute to the increased expression of interleukin-6 in brain of aged mice," NeuroImmunoModulation, vol. 9, no. 4, pp. 183-192, 2001.

[49] N. R. Nichols, J. R. Day, N. J. Laping, S. A. Johnson, and C. E. Finch, "GFAP mRNA increases with age in rat and human brain," Neurobiology of Aging, vol. 14, no. 5, pp. 421-429, 1993.

[50] M. L. Cotrina and M. Nedergaard, "Peroxynitrite triggers a phenotypic transformation in spinal cord astrocytes that induces motor neuron apoptosis," Journal of Neuroscience Research, vol. 67, no. 1, pp. 21-29, 2002.

[51] R. Porchet, A. Probst, C. Bouras, E. Dráberová, P. Dráber, and B. M. Riederer, "Analysis of gial acidic fibrillary protein in the human entorhinal cortex during aging and in Alzheimer's disease," Proteomics, vol. 3, no. 8, pp. 1476-1485, 2003.

[52] M. Pekny and M. Pekna, "Astrocyte intermediate filaments in CNS pathologies and regeneration," Journal of Pathology, vol. 204, no. 4, pp. 428-437, 2004.

[53] J. Tichauer, K. Saud, and R. von Bernhardi, "Modulation by astrocytes of microglial cell-mediated neuroinflammation: effect on the activation of microglial signaling pathways," NeuroImmunoModulation, vol. 14, no. 3-4, pp. 168-174, 2007.
[54] N. J. Abbott, L. Rönnbäck, and E. Hansson, "Astrocyteendothelial interactions at the blood-brain barrier," Nature Reviews Neuroscience, vol. 7, no. 1, pp. 41-53, 2006.

[55] B. V. Zlokovic, "The blood-brain barrier in health and chronic neurodegenerative disorders," Neuron, vol. 57, no. 2, pp. 178-201, 2008.

[56] J. M. Weiss and J. W. Berman, "Astrocyte expression of monocyte chemoattractant protein-1 is differentially regulated by transforming growth factor beta," Journal of Neuroimmunology, vol. 91, no. 1-2, pp. 190-197, 1998.

[57] Y. Couch, L. Alvarez-Erviti, N. R. Sibson, M. J. A. Wood, and D. C. Anthony, "The acute inflammatory response to intranigral $\alpha$ synuclein differs significantly from intranigral lipopolysaccharide and is exacerbated by peripheral inflammation," Journal of Neuroinflammation, vol. 8, article 166, 2011.

[58] A. D. Reynolds, D. K. Stone, R. L. Mosley, and H. E. Gendelman, "Proteomic studies of nitrated alpha-synuclein microglia regulation by CD4+CD25+ T cells," Journal of Proteome Research, vol. 8, no. 7, pp. 3497-3511, 2009.

[59] A. D. Reynolds, D. K. Stone, R. L. Mosley, and H. E. Gendelman, "Nitrated $\alpha$-synuclein-induced alterations in microglial immunity are regulated by CD4+ T cell subsets," Journal of Immunology, vol. 182, no. 7, pp. 4137-4149, 2009.

[60] D. Sulzer, "Multiple hit hypotheses for dopamine neuron loss in Parkinson's disease," Trends in Neurosciences, vol. 30, no. 5, pp. 244-250, 2007.

[61] H.-J. Lee, S. Patel, and S.-J. Lee, "Intravesicular localization and exocytosis of $\alpha$-synuclein and its aggregates," Journal of Neuroscience, vol. 25, no. 25, pp. 6016-6024, 2005.

[62] J. Jin, F.-S. Shie, J. Liu et al., "Prostaglandin E2 receptor subtype 2 (EP2) regulates microglial activation and associated neurotoxicity induced by aggregated $\alpha$-synuclein," Journal of Neuroinflammation, vol. 4, article 2, 2007.

[63] T. Maekawa, M. Kubo, I. Yokoyama, E. Ohta, and F. Obata, "Agedependent and cell-population-restricted LRRK2 expression in normal mouse spleen," Biochemical and Biophysical Research Communications, vol. 392, no. 3, pp. 431-435, 2010.

[64] J. Miklossy, T. Arai, J.-P. Guo et al., "LRRK2 expression in normal and pathologic human brain and in human cell lines," Journal of Neuropathology and Experimental Neurology, vol. 65, no. 10, pp. 953-963, 2006.

[65] F. Gillardon, R. Schmid, and H. Draheim, “Parkinson's diseaselinked leucine-rich repeat kinase 2 (R1441G) mutation increases proinflammatory cytokine release from activated primary microglial cells and resultant neurotoxicity," Neuroscience, vol. 208, pp. 41-48, 2012.

[66] R. Lopez de Maturana, J. C. Aguila, A. Sousa et al., "Leucinerich repeat kinase 2 modulates cyclooxygenase 2 and the inflammatory response in idiopathic and genetic Parkinson's disease," Neurobiology of Aging, vol. 35, no. 5, pp. 1116-1124, 2014.

[67] D. F. Marker, J. M. Puccini, T. E. Mockus, J. Barbieri, S. M. $\mathrm{Lu}$, and H. A. Gelbard, "LRRK2 kinase inhibition prevents pathological microglial phagocytosis in response to HIV-1 Tat protein," Journal of Neuroinflammation, vol. 9, article 261, 2012.

[68] B. Kim, M.-S. Yang, D. Choi et al., "Impaired inflammatory responses in murine lrrk2-knockdown brain microglia," PLoS ONE, vol. 7, no. 4, Article ID e34693, 2012.

[69] H. Shimura, N. Hattori, S.-I. Kubo et al., "Familial Parkinson disease gene product, parkin, is a ubiquitin-protein ligase," Nature Genetics, vol. 25, no. 3, pp. 302-305, 2000. 
[70] T. C. Frank-Cannon, T. Tran, K. A. Ruhn et al., "Parkin deficiency increases vulnerability to inflammation-related nigral degeneration," Journal of Neuroscience, vol. 28, no. 43, pp. 10825-10834, 2008.

[71] B. Zheng, Z. Liao, J. J. Locascio et al., "PGC-1alpha, a potential therapeutic target for early intervention in Parkinson's disease," Science Translational Medicine, vol. 2, no. 52, Article ID 52ra73, 2010.

[72] J. St-Pierre, S. Drori, M. Uldry et al., "Suppression of reactive oxygen species and neurodegeneration by the PGC-1 transcriptional coactivators," Cell, vol. 127, no. 2, pp. 397-408, 2006.

[73] G. Mudò, J. Mäkelä, V. Di Liberto et al., “Transgenic expression and activation of PGC- $1 \alpha$ protect dopaminergic neurons in the MPTP mouse model of Parkinsons disease," Cellular and Molecular Life Sciences, vol. 69, no. 7, pp. 1153-1165, 2012.

[74] S. Sczelecki, A. Besse-Patin, A. Abboud et al., "Loss of Pgclalpha expression in aging mouse muscle potentiates glucose intolerance and systemic inflammation," The American Journal of Physiology Endocrinology and Metabolism, vol. 306, no. 2, pp. E157-E167, 2014.

[75] P. Scalzo, A. Kümmer, F. Cardoso, and A. L. Teixeira, "Increased serum levels of soluble tumor necrosis factor- $\alpha$ receptor-1 in patients with Parkinson's disease," Journal of Neuroimmunology, vol. 216, no. 1-2, pp. 122-125, 2009.

[76] M. Dufek, M. Hamanová, J. Lokaj et al., "Serum inflammatory biomarkers in Parkinson's disease," Parkinsonism and Related Disorders, vol. 15, no. 4, pp. 318-320, 2009.

[77] H. Chen, E. J. O’Reilly, M. A. Schwarzschild, and A. Ascherio, "Peripheral inflammatory biomarkers and risk of Parkinson's disease," The American Journal of Epidemiology, vol. 167, no. 1, pp. 90-95, 2008.

[78] D. Devos, T. Lebouvier, B. Lardeux et al., "Colonic inflammation in Parkinson's disease," Neurobiology of Disease, vol. 50, pp. 4248, 2013.

[79] H. Przuntek, T. Müller, and P. Riederer, "Diagnostic staging of Parkinson's disease: conceptual aspects," Journal of Neural Transmission, vol. 111, no. 2, pp. 201-216, 2004.

[80] C. Weller, N. Oxlade, S. M. Dobbs, R. J. Dobbs, A. Charlett, and I. T. Bjarnason, "Role of inflammation in gastrointestinal tract in aetiology and pathogenesis of idiopathic parkinsonism," FEMS Immunology and Medical Microbiology, vol. 44, no. 2, pp. 129-135, 2005.

[81] Y. Hasegawa, T. Inagaki, M. Sawada, and A. Suzumura, "Impaired cytokine production by peripheral blood mononuclear cells and monocytes/macrophages in Parkinson's disease," Acta Neurologica Scandinavica, vol. 101, no. 3, pp. 159-164, 2000.

[82] M. K. Beyer, K. Herlofson, D. Arsland, and J. P. Larsen, "Causes of death in a community-based study of Parkinson's disease," Acta Neurologica Scandinavica, vol. 103, no. 1, pp. 7-11, 2001.

[83] M. D’Amelio, P. Ragonese, L. Morgante et al., "Long-term survival of Parkinson's disease: a population-based study," Journal of Neurology, vol. 253, no. 1, pp. 33-37, 2006.

[84] H. Arai, T. Furuya, Y. Mizuno, and H. Mochizuki, "Inflammation and infection in Parkinson's disease," Histology and Histopathology, vol. 21, no. 4-6, pp. 673-678, 2006.

[85] V. Brochard, B. Combadière, A. Prigent et al., "Infiltration of CD4+ lymphocytes into the brain contributes to neurodegeneration in a mouse model of Parkinson disease," Journal of Clinical Investigation, vol. 119, no. 1, pp. 182-192, 2009.
[86] D. K. Stone, A. D. Reynolds, R. L. Mosley, and H. E. Gendelman, "Innate and adaptive immunity for the pathobiology of Parkinson's disease," Antioxidants and Redox Signaling, vol. 11, no. 9, pp. 2151-2166, 2009.

[87] R. Kortekaas, K. L. Leenders, J. C. H. van Oostrom et al., "Bloodbrain barrier dysfunction in Parkinsonian midbrain in vivo," Annals of Neurology, vol. 57, no. 2, pp. 176-179, 2005.

[88] E. C. Hirsch and S. Hunot, "Neuroinflammation in Parkinson's disease: a target for neuroprotection?" The Lancet Neurology, vol. 8, no. 4, pp. 382-397, 2009.

[89] P. M. Carvey, Q. Chang, J. W. Lipton, and Z. Ling, "Prenatal exposure to the bacteriotoxin lipopolysaccharide leads to longterm losses of dopamine neurons in offspring: a potential, new model of Parkinson's disease," Frontiers in Bioscience, vol. 8, pp. s826-s837, 2003.

[90] Z. Ling, D. A. Gayle, S. Y. Ma et al., "In utero bacterial endotoxin exposure causes loss of tyrosine hydroxylase neurons in the postnatal rat midbrain," Movement Disorders, vol. 17, no. 1, pp. 116-124, 2002.

[91] Z. Ling, Q. A. Chang, C. W. Tong, S. E. Leurgans, J. W. Lipton, and P. M. Carvey, "Rotenone potentiates dopamine neuron loss in animals exposed to lipopolysaccharide prenatally," Experimental Neurology, vol. 190, no. 2, pp. 373-383, 2004.

[92] H. Engler, R. Doenlen, C. Riether et al., "Time-dependent alterations of peripheral immune parameters after nigrostriatal dopamine depletion in a rat model of Parkinson's disease," Brain, Behavior, and Immunity, vol. 23, no. 4, pp. 518-526, 2009.

[93] R. F. Villarán, A. M. Espinosa-Oliva, M. Sarmiento et al., "Ulcerative colitis exacerbates lipopolysaccharide-induced damage to the nigral dopaminergic system: potential risk factor in Parkinson's disease," Journal of Neurochemistry, vol. 114, no. 6, pp. 1687-1700, 2010.

[94] C. H. Hawkes, K. Del Tredici, and H. Braak, "A timeline for Parkinson's disease," Parkinsonism and Related Disorders, vol. 16, no. 2, pp. 79-84, 2010.

[95] M. Menza, R. D. Dobkin, H. Marin et al., "The role of inflammatory cytokines in cognition and other non-motor symptoms of Parkinson's disease," Psychosomatics, vol. 51, no. 6, pp. 474-479, 2010.

[96] M. J. Blaser, "Who are we? Indigenous microbes and the ecology of human diseases," EMBO Reports, vol. 7, no. 10, pp. 956-960, 2006.

[97] H. H. Nielsen, J. Qiu, S. Friis, L. Wermuth, and B. Ritz, “Treatment for Helicobacter pylori infection and risk of parkinson's disease in Denmark," European Journal of Neurology, vol. 19, no. 6, pp. 864-869, 2012.

[98] I. T. Bjarnason, A. Charlett, R. J. Dobbs et al., "Role of chronic infection and inflammation in the gastrointestinal tract in the etiology and pathogenesis of idiopathic Parkinsonism. Part 2: response of facets of clinical idiopathic Parkinsonism to Helicobacter pylori eradication. A randomized, double-blind, placebo-controlled efficacy study," Helicobacter, vol. 10, pp. 557287, 2005.

[99] R. Savica, J. M. Carlin, B. R. Grossardt et al., "Medical records documentation of constipation preceding Parkinson disease: a case-control study," Neurology, vol. 73, no. 21, pp. 1752-1758, 2009.

[100] Z. Arvanitakis, R. S. Wilson, J. A. Schneider, J. L. Bienias, D. A. Evans, and D. A. Bennett, "Diabetes mellitus and progression of rigidity and gait disturbance in older persons," Neurology, vol. 63, no. 6, pp. 996-1001, 2004. 
[101] S. E. Shoelson, J. Lee, and A. B. Goldfine, "Inflammation and insulin resistance," Journal of Clinical Investigation, vol. 116, no. 7, pp. 1793-1801, 2006.

[102] A. H. Berg and P. E. Scherer, "Adipose tissue, inflammation, and cardiovascular disease," Circulation Research, vol. 96, no. 9, pp. 939-949, 2005.

[103] C. Zoccali, G. Tripepi, F. Cambareri et al., "Adipose tissue cytokines, insulin sensitivity, inflammation, and cardiovascular outcomes in end-stage renal disease patients," Journal of Renal Nutrition, vol. 15, no. 1, pp. 125-130, 2005.

[104] D. Cai, M. Yuan, D. F. Frantz et al., "Local and systemic insulin resistance resulting from hepatic activation of IKK- $\beta$ and NF$\kappa$ B," Nature Medicine, vol. 11, no. 2, pp. 183-190, 2005.

[105] J. A. Ehses, A. Perren, E. Eppler et al., "Increased number of islet-associated macrophages in type 2 diabetes," Diabetes, vol. 56, no. 9, pp. 2356-2370, 2007.

[106] C. T. De Souza, E. P. Araujo, S. Bordin et al., "Consumption of a fat-rich diet activates a proinflammatory response and induces insulin resistance in the hypothalamus," Endocrinology, vol. 146, no. 10, pp. 4192-4199, 2005.

[107] M. Saghizadeh, J. M. Ong, W. T. Garvey, R. R. Henry, and P. A. Kern, "The expression of TNF $\alpha$ by human muscle: relationship to insulin resistance," Journal of Clinical Investigation, vol. 97, no. 4, pp. 1111-1116, 1996.

[108] C. J. Barnum and M. G. Tansey, "Neuroinflammation and nonmotor symptoms: the dark passenger of Parkinson's disease?" Current Neurology and Neuroscience Reports, vol. 12, no. 4, pp. 350-358, 2012.

[109] H. B. Stolp and K. M. Dziegielewska, "Review: role of developmental inflammation and blood-brain barrier dysfunction in neurodevelopmental and neurodegenerative diseases," Neuropathology and Applied Neurobiology, vol. 35, no. 2, pp. 132-146, 2009.

[110] K. J. Tracey, "The inflammatory reflex," Nature, vol. 420, no. 6917, pp. 853-859, 2002.

[111] E. C. Hirsch, S. Vyas, and S. Hunot, "Neuroinflammation in Parkinson's disease," Parkinsonism and Related Disorders, vol. 18, no. 1, pp. S210-S212, 2012.

[112] N. Laflamme, S. Lacroix, and S. Rivest, "An essential role of interleukin- $1 \beta$ in mediating NF- $\kappa \mathrm{B}$ activity and COX-2 transcription in cells of the blood-brain barrier in response to a systemic and localized inflammation but not during endotoxemia," Journal of Neuroscience, vol. 19, no. 24, pp. 1092310930, 1999.

[113] C. M. Blatteis, "Role of the OVLT in the febrile response to circulating pyrogens," Progress in Brain Research, vol. 91, pp. 409-412, 1992.

[114] C. J. Henry, Y. Huang, A. M. Wynne, and J. P. Godbout, "Peripheral lipopolysaccharide (LPS) challenge promotes microglial hyperactivity in aged mice that is associated with exaggerated induction of both pro-inflammatory IL- $1 \beta$ and antiinflammatory IL-10 cytokines," Brain, Behavior, and Immunity, vol. 23, no. 3, pp. 309-317, 2009.

[115] H.-M. Gao, F. Zhang, H. Zhou, W. Kam, B. Wilson, and J.S. Hong, "Neuroinflammation and $\alpha$-synuclein dysfunction potentiate each other, driving chronic progression of neurodegeneration in a mouse model of Parkinson's disease," Environmental Health Perspectives, vol. 119, no. 6, pp. 807-814, 2011.

[116] T. G. Ton, S. R. Heckbert, W. T. Longstreth Jr. et al., "Nonsteroidal anti-inflammatory drugs and risk of Parkinson's disease," Movement Disorders, vol. 21, no. 7, pp. 964-969, 2006.
[117] J. M. Plane, Y. Shen, D. E. Pleasure, and W. Deng, "Prospects for minocycline neuroprotection," Archives of Neurology, vol. 67, no. 12, pp. 1442-1448, 2010.

[118] B. Ravina, "A randomized, double-blind, futility clinical trial of creatine and minocycline in early Parkinson disease," Neurology, vol. 66, no. 5, pp. 664-671, 2006.

[119] J. Thévenet, R. Pescini Gobert, R. Hooft van Huijsduijnen, C. Wiessner, and Y. J. Sagot, "Regulation of LRRK2 expression points to a functional role in human monocyte maturation," PLoS ONE, vol. 6, no. 6, Article ID e21519, 2011.

[120] M. S. Moehle, P. J. Webber, T. Tse et al., "LRRK2 inhibition attenuates microglial inflammatory responses," Journal of Neuroscience, vol. 32, no. 5, pp. 1602-1611, 2012.

[121] K. M. Hinkle, M. Yue, B. Behrouz et al., "LRRK2 knockout mice have an intact dopaminergic system but display alterations in exploratory and motor co-ordination behaviors," Molecular Neurodegeneration, vol. 7, article 25, 2012.

[122] T. Wenz, "PGC-1 $\alpha$ activation as a therapeutic approach in mitochondrial disease," IUBMB Life, vol. 61, no. 11, pp. 10511062,2009

[123] E. Hondares, I. Pineda-Torra, R. Iglesias, B. Staels, F. Villarroya, and M. Giralt, "PPAR $\delta$, but not PPAR $\alpha$, activates PGC- $1 \alpha$ gene transcription in muscle," Biochemical and Biophysical Research Communications, vol. 354, no. 4, pp. 1021-1027, 2007.

[124] R. Pardo, N. Enguix, J. Lasheras, J. E. Feliu, A. Kralli, and J. A. Villena, "Rosiglitazone-induced mitochondrial biogenesis in white adipose tissue is independent of peroxisome proliferatoractivated receptor $\gamma$ coactivator- $1 \alpha$, , PLoS ONE, vol. 6, no. 11, Article ID e26989, 2011.

[125] M. Suwa, T. Egashira, H. Nakano, H. Sasaki, and S. Kumagai, "Metformin increases the PGC- $1 \alpha$ protein and oxidative enzyme activities possibly via AMPK phosphorylation in skeletal muscle in vivo," Journal of Applied Physiology, vol. 101, no. 6, pp. 1685-1692, 2006.

[126] W. Chowanadisai, K. A. Bauerly, E. Tchaparian, A. Wong, G. A. Cortopassi, and R. B. Rucker, "Pyrroloquinoline quinone stimulates mitochondrial biogenesis through cAMP response element-binding protein phosphorylation and increased PGC$1 \alpha$ expression," Journal of Biological Chemistry, vol. 285, no. 1 , pp. 142-152, 2010.

[127] I. Irrcher, V. Ljubicic, A. F. Kirwan, and D. A. Hood, "AMPactivated protein kinase-regulated activation of the PGC-lalpha promoter in skeletal muscle cells," PLoS ONE, vol. 3, no. 10, Article ID e3614, 2008.

[128] M. Lagouge, C. Argmann, Z. Gerhart-Hines et al., "Resveratrol Improves Mitochondrial Function and Protects against Metabolic Disease by Activating SIRT1 and PGC-1 $\alpha$," Cell, vol. 127, no. 6, pp. 1109-1122, 2006. 

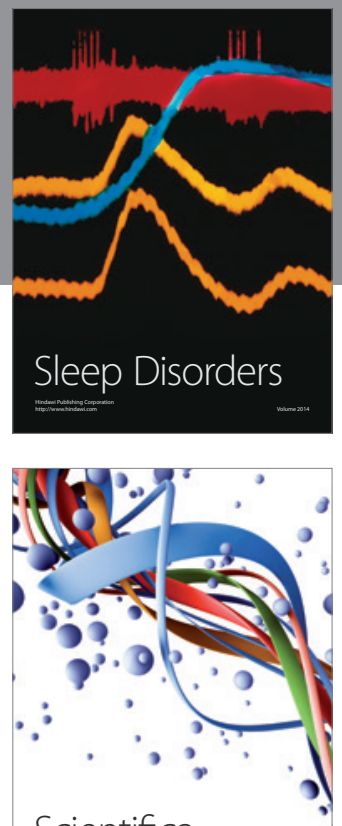

Scientifica
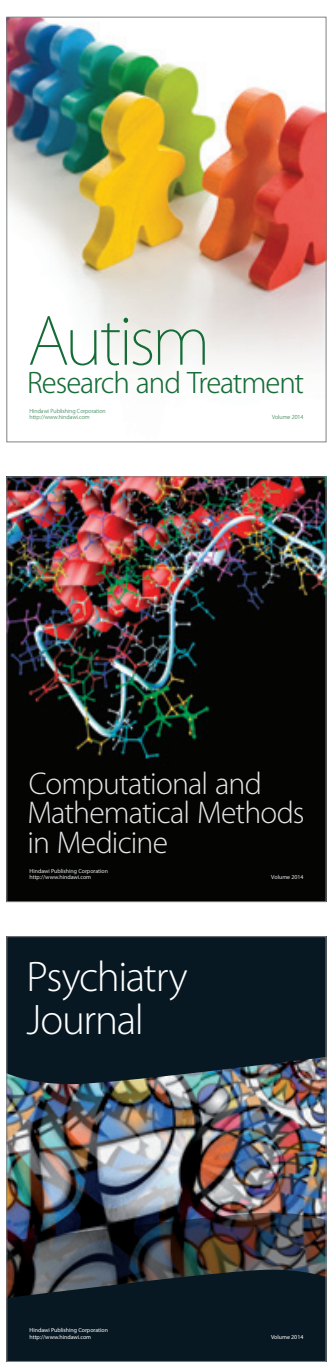
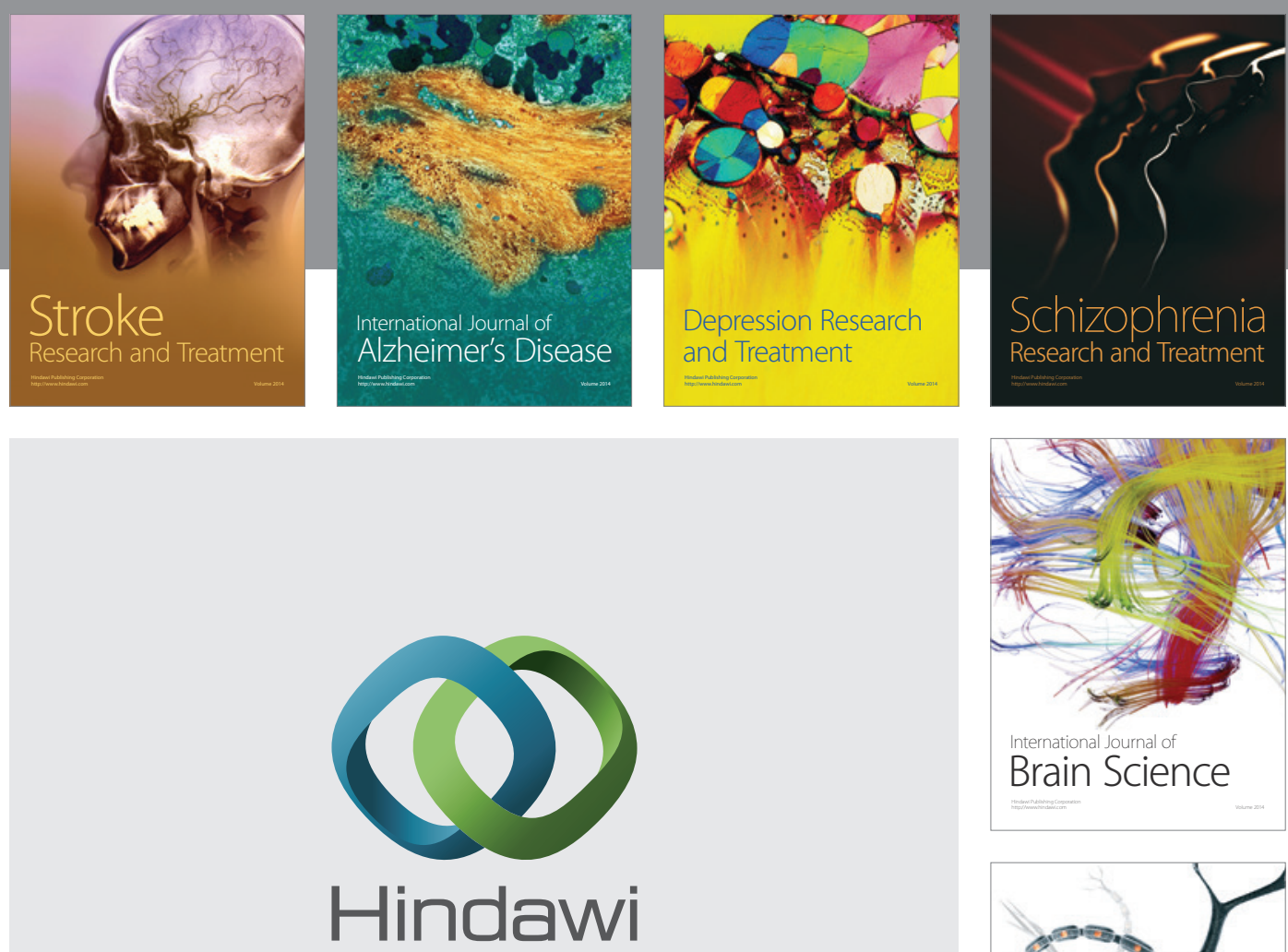

Submit your manuscripts at

http://www.hindawi.com
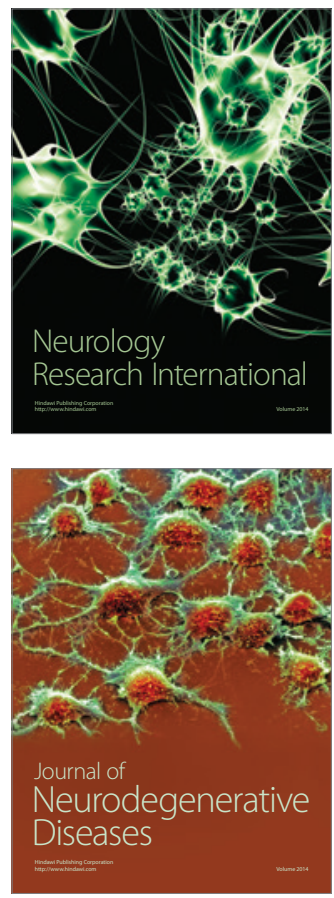

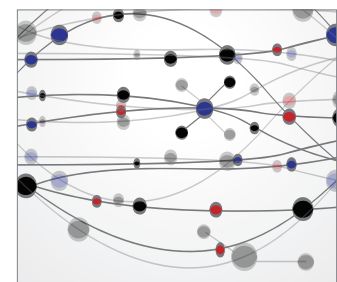

The Scientific World Journal
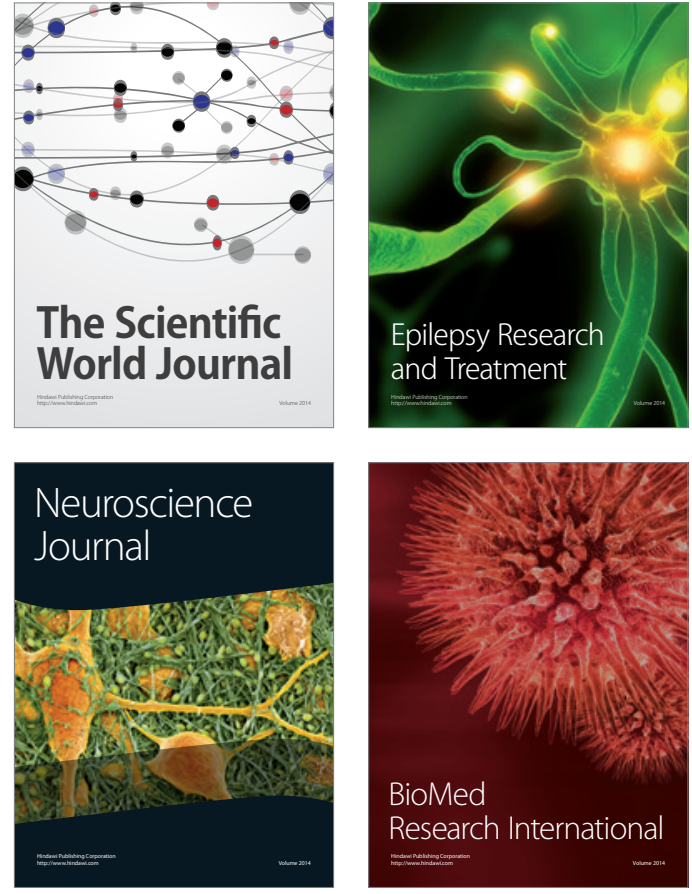

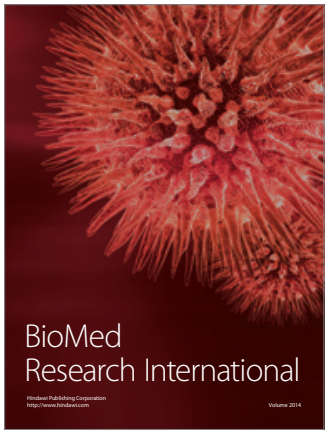

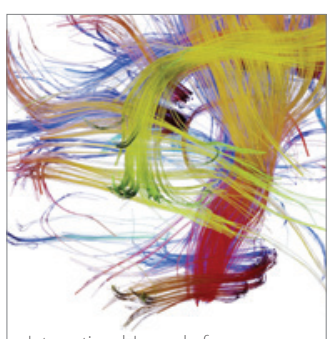

Brain Science

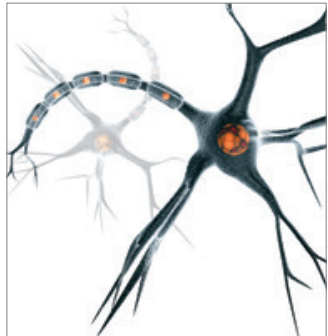

Neural Plasticity
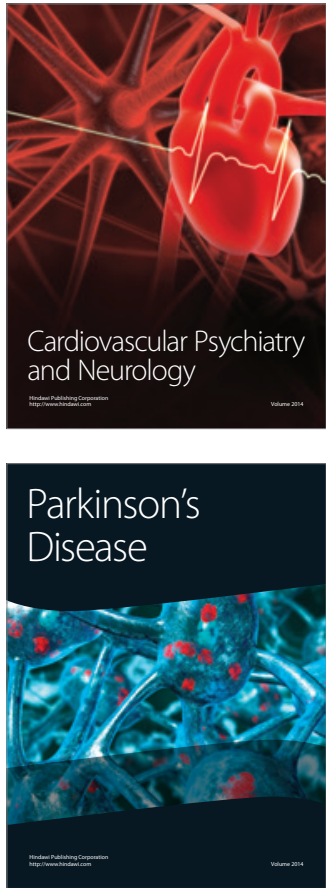